\title{
Multiple Electron Diffraction and Two-Dimensional Crystalline Order in Liquid-Crystal Thin Films
}

\author{
Chih-Yu Chao, ${ }^{1, *}$ Tung-Cheng Pan, ${ }^{1}$ Chia-Fu Chou, ${ }^{2, \dagger}$ and John T. $\mathrm{Ho}^{2}$ \\ ${ }^{1}$ Department of Physics, National Taiwan University, Taipei 10617, Taiwan, Republic of China \\ ${ }^{2}$ Department of Physics, University at Buffalo, The State University of New York, Buffalo, New York 14260, USA
}

(Received 9 January 2003; published 19 September 2003)

Electron diffraction in freestanding thin films in the crystal- $B$ phase reveals extensive multiple diffraction in films of 14S5 but not of 4O.8, suggesting an important difference in the structural order in these films despite the similarities in their positional correlations found in earlier experiments. The result in a two-layer 14S5 film indicates surprisingly that secondary diffraction can occur in a crystal with only pseudo-long-range positional order.

DOI: 10.1103/PhysRevLett.91.125504

PACS numbers: 61.30.- $-\mathrm{v}, 61.14 .-\mathrm{x}$

It is well known that a two-dimensional (2D) solid, unlike its three-dimensional counterpart, is not expected to exhibit true long-range positional order because of the logarithmic divergence of the mean-square fluctuations of the interatomic separation $r$ as $r$ diverges to infinity [1-3]. On the other hand, the positional correlations in a 2D solid, because of its resistance to shear, is expected to decay algebraically as $r^{-\eta}$, in contrast to the short-range exponential decay in a 2D liquid [4-6]. The exponent $\eta$ is related to the compression and shear moduli. One possible indicator of the existence of this pseudo-longrange algebraic positional order in a $2 \mathrm{D}$ solid is the scattering line shape $S(\boldsymbol{Q})$ at the Bragg positions $\boldsymbol{G}$ as a function of the scattering wave vector $\boldsymbol{Q}$, which is expected to have the form $|\boldsymbol{Q}-\boldsymbol{G}|^{-2+\eta}[5,7]$. Perhaps the most promising system in which to study 2D crystalline order is the freestanding smectic liquidcrystal film, which is free from the influence of a substrate. The predicted scattering line shape has been verified in $\mathrm{x}$-ray diffraction experiments on freestanding liquid-crystal thin films in the crystal- $B$ $($ Cry- $B)$ phase $[8,9]$. The value of $\eta$ was found to be 0.13 in a two-layer film of 4-n-pentylbenzenethio- $4^{\prime}-n$ tetradecyloxybenzoate (14S5) [8] and 0.15 in a 4-layer film of $N$-(4-n-butyloxybenzylidene)- $4^{\prime}$ - $n$-octylaniline (40.8) [9]. Another study on a different material revealed the expected decrease of $\eta$ as the thickness increases because of dimensional crossover [10].

The nature of the Cry- $B$ phase itself, which is generally believed to consist of weakly coupled crystalline layers leading to three-dimensional positional order, is also of considerable interest. Measurements using torsional oscillators have shown that Cry- $B$ films of $14 \mathrm{~S} 5$ and 40.8 exhibit in-plane shear responses [11,12]. The shear elastic modulus $\mathrm{C}_{44}$ in the bulk Cry- $B$ phase of 40.8 was found to be $1.2 \times 10^{5} \mathrm{~N} / \mathrm{m}^{2}$ [13], which is relatively small compared to ordinary solids. Furthermore, the interlayer disorder in freestanding Cry- $B$ films of 40.8 has been shown by $\mathrm{x}$-ray scattering to be similar to that in the more fluid smectic- $A$ (Sm- $A)$ films [14]. In addition, electron diffraction has revealed that a freestanding $\mathrm{Sm}-A$ film of 40.8 freezes into a Cry- $B$ film through layer-bylayer transitions involving the intermediate hexatic- $B$ $($ Hex- $B)$ phase [15], while a 14S5 film undergoes layerby-layer Sm- $A-\mathrm{Cry}-B$ transitions without involving the Hex- $B$ phase [16], suggesting stronger interlayer coupling in $14 \mathrm{~S} 5$ than in $4 \mathrm{O} .8$.

We report here the novel use of electron diffraction to examine $2 \mathrm{D}$ crystalline order in ultrathin Cry- $B$ films. Unlike $\mathrm{x}$ ray, electrons interact strongly with matter, leading to the possibility of incoherent secondary or even tertiary scattering if the diffracting medium is essentially a perfect crystal [17-19]. Thus multiple electron diffraction could be used as an alternative indicator of the degree of crystalline order in the 2D limit. We have studied both 14 S5 and 4O.8, whose Cry- $B$ thin films were found previously to give similar $\mathrm{x}$-ray diffraction line shapes consistent with quasi-long-range algebraic positional correlations $[8,9]$. We find that $14 \mathrm{~S} 5$, but not $4 \mathrm{O} .8$, shows strong multiple electron diffraction in thin films suggestive of a high degree of crystalline order. Our result in a two-layer 14S5 Cry- $B$ film indicates surprisingly that secondary diffraction can nevertheless occur in a crystal with only pseudo-long-range positional order. The difference in diffraction in the Cry- $B$ phase between $14 \mathrm{~S} 5$ and 40.8 reveals that different degrees of interlayer positional ordering can occur among Cry- $B$ phases in different materials.

Our experimental technique for making electrondiffraction measurements of freestanding liquid-crystal thin films using a transmission electron microscope equipped with a pressurized, temperature-controlled sample chamber has been reported elsewhere [20]. We studied freestanding smectic films from 2 to 20 molecular layers in thickness suspended across an aperture $1 \mathrm{~mm}$ in diameter. At high temperatures, 14S5 and 4O.8 exhibit the $\mathrm{Sm}-A$ phase, composed of liquidlike layers with the long axis of the molecules normal to them. In the bulk, 
both materials freeze directly from the $\mathrm{Sm}-A$ to the orthogonal Cry- $B$ phase, at $66.5^{\circ} \mathrm{C}$ for $14 \mathrm{~S} 5$ [12] and $49^{\circ} \mathrm{C}$ for 40.8 [8]. We concentrated our effort in this study on electron diffraction in films of both materials that were entirely in the Cry- $B$ phase.

We first present the data on 14S5. The electrondiffraction pattern shown in Fig. 1 for a six-layer film represents a typical result obtained in 14S5 films of more than two molecular layers in the Cry- $B$ phase below $66.5^{\circ} \mathrm{C}$. While the diffraction pattern is unusually complex, several clear features are immediately apparent. The first is the occurrence of six strong spots arranged at $60^{\circ}$ intervals along a circle of radius $q$ 。 surrounding the incident beam, indicative of the lowest-order diffraction of the primary beam by a single hexagonal crystalline domain. The second is the existence of three weaker but still prominent spots arranged in an arc of about $10^{\circ}$ along the circle on one side of each of the six strong diffraction spots. Because these spots lie on the primary circle centered around the central incident-beam spot, they represent primary diffraction by smaller domains present within the illuminated area whose crystalline axes deviate from one another by about $3^{\circ}-10^{\circ}$. The indexing of the primary diffraction spots from the multicrystalline film is also shown in Fig. 1. The third feature is the presence of other even weaker spots surrounding each strong diffraction spot, in groups of three along arcs in five different directions. The origins of the extra diffraction spots are indicated in Fig. 2 for the groups surrounding the $\overline{1} 10$ primary spots. The spots along the five arcs can be separated into two categories. Those along the two arcs closest to the $\overline{1} 10$ primary spots are the result of secondary diffraction, by an interior layer of

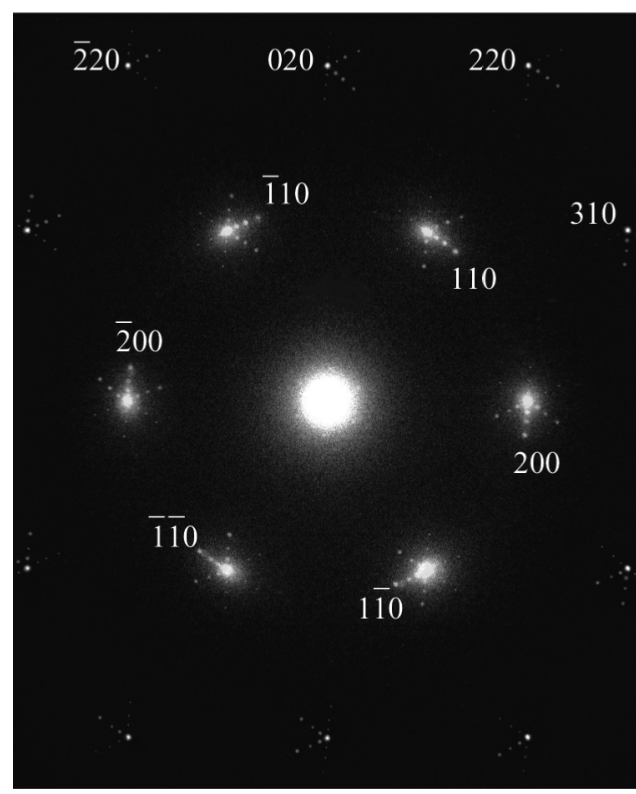

FIG. 1. Electron-diffraction pattern of a six-layer 14S5 film at $64.5^{\circ} \mathrm{C}$ and indexing of some primary diffraction spots. the film, of electrons which have been diffracted once by the surface layer. For example, using conventional notations [17,18], the spots labeled $110+\overline{2} 00$ are the result of 110 primary diffraction followed by $\overline{2} 00$ secondary diffraction. This identification can be seen by considering each one of the 110 spots as an incident beam, drawing a circle of radius $q$ 。 around it, and finding that the circle intersects six secondary spots that are weaker in intensity than the primary spots, with the $110+\overline{2} 00$ spot being the one to the left of the 110 spot. The spots along the remaining three arcs adjacent to each set of primary spots are the result of tertiary diffraction, by an even deeper layer of the film, of electrons that have already undergone both primary and secondary diffraction. For example, those labeled $200+\overline{1} 10+\overline{2} 00$ are the result of $\overline{2} 00$ tertiary diffraction following first 200 primary diffraction and then $\overline{1} 10$ secondary diffraction. Again, this identification can be understood by considering each of the secondary spots as incident beam and locating the six tertiary spots that lie on a circle of radius $q$ 。 around it. Our interpretation of the secondary and tertiary spots is unambiguous, as it is the only one that can account for the relative positions of various domain spots. It is also reinforced by the fact that the tertiary spots are weaker than the secondary spots, which in turn are weaker than the primary spots. The unusual observance of tertiary electron diffraction is unambiguously identified here only because of the fortuitous presence of the slightly disorientated domains in the illuminated region. Since the probing electron beam has a diameter of $50 \mu \mathrm{m}$, we estimate that the illuminated region typically contains a main crystalline domain of 30 to $40 \mu \mathrm{m}$ in size and several smaller, slightly disorientated, domains of 3 to $10 \mu \mathrm{m}$. More importantly, the existence of tertiary electron diffraction suggests a very high degree of positional order both within and among the molecular layers in the six-layer 14S5 Cry- $B$ film.

Electron-diffraction patterns similar to Fig. 1 were obtained in other 14S5 Cry- $B$ films of at least three molecular layers. On the other hand, the electrondiffraction pattern in a two-layer $14 \mathrm{~S} 5 \mathrm{Cry}-B$ film is

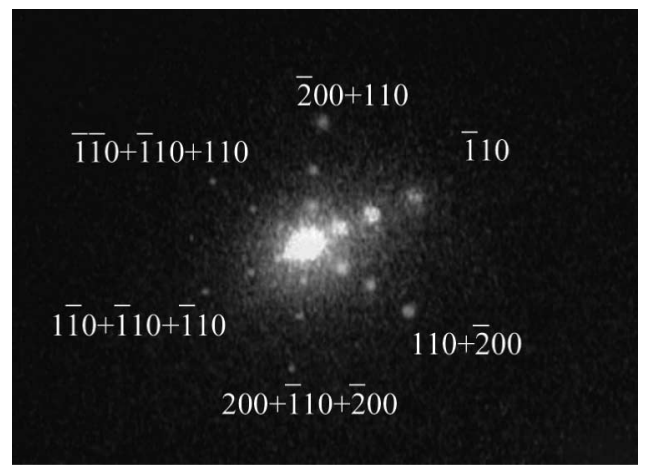

FIG. 2. Indexing of a cluster of multiple diffraction spots in Fig. 1. 
distinctly different, as shown in Fig. 3. Each of the six strongest diffraction spots forming a hexagonal pattern is accompanied by six weaker spots on either side of it spread over a mosaic arc of about $20^{\circ}$ along the main circle. These spots are indicative of primary diffraction in a multicrystalline film. In addition, surrounding each strong spot are even weaker spots lying along arcs in two other directions. These are identified as originating from secondary diffraction by the lower layer of the film of electrons that have undergone primary diffraction by the upper layer. The indexing of selected primary and secondary diffraction spots from the two-layer film is also shown in Fig. 3. There is no evidence of tertiary diffraction in the two-layer film. The fact that tertiary diffraction is observed in films of three or more layers but not in the two-layer film further reinforces our interpretation of the diffraction spots, and is also a good indication of the uniformity in thickness of the two-layer film.

We now turn to the data on 40.8. All the 40.8 Cry- $B$ films with two or more layers that we have examined gave electron-diffraction patterns similar to the one shown in Fig. 4. The dominant feature is a hexagonal array of six strong spots indicative of lower-order primary diffraction by a single crystal. There is no evidence of multicrystalline domains, nor of higher-order primary diffraction, even in overexposed patterns. The much weaker higherorder diffraction spots in 40.8 compared to $14 \mathrm{~S} 5$ are probably due to the different structure factors of these two materials with considerably different molecular structures [17]. The lack of multicrystalline diffraction makes it difficult to completely rule out the presence of multiple diffraction, but the absence of diffraction spots at the higher-order locations makes it highly unlikely.

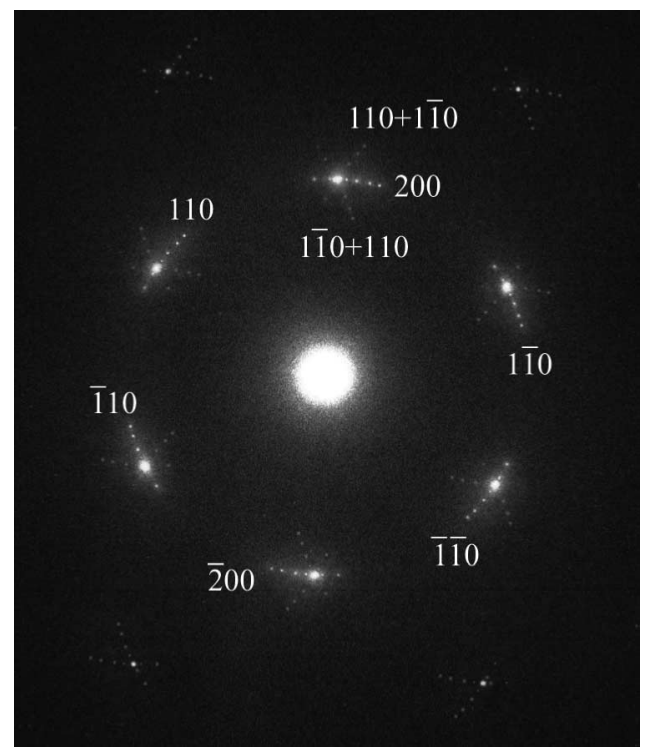

FIG. 3. Electron-diffraction pattern of a two-layer 14S5 film at $71.5^{\circ} \mathrm{C}$ and indexing of some primary and secondary diffraction spots.
Our observation of multiple electron diffraction in Cry- $B$ films of 14 S5 but not in 40.8 leads to the important conclusion that different degrees of crystallinity can occur among Cry- $B$ phases in different materials. In particular, the interlayer positional order in the Cry- $B$ phase in 14S5 appears to be much stronger than that in 4O.8. It should be pointed out that several earlier experimental results may be directly relevant to this conclusion. First, electron diffraction on these two materials has shown different surface-induced layer-by-layer transitions from the Sm- $A$ to the Cry- $B$ phase $[15,16]$. The fact that the intermediate Hex- $B$ phase, which is predicted to occur in two dimensions [21,22], is involved in the surface freezing in 40.8 but not in 14S5 suggests that the smectic layers in 40.8 are more weakly coupled and thus more 2D-like than in 14S5, which is consistent with our current observation of stronger interlayer ordering in the Cry- $B$ phase in 14 S5 than in 40.8 . Second, the absence of secondary diffraction in the Cry- $B$ phase in 40.8 films is also consistent with the occurrence of Sm- $A$-like layer fluctuations in these films found in $\mathrm{x}$-ray scattering [14]. It has been suggested that the relatively large fluctuations in a 40.8 Cry- $B$ film are due to the fact that the enhancement of the effective surface tension by the shear modulus $\mathrm{C}_{44}$ is small in 40.8 unless the film is very thick [23]. Third, the random stacking of the layer-by-layer positional order found in another x-ray study of 40.8 Cry- $B$ films [24] may be another manifestation of the interlayer disorder. Fourth, a Raman study also revealed that 40.8 in the Cry- $B$ phase already exhibits fluidlike dynamics in its central molecular core similar to the $\mathrm{Sm}-A$ phase and distinct from the solid phase [25]. The cumulative evidence all bolsters our current result, which suggests much less interlayer disorder and a considerably larger $\mathrm{C}_{44}$ in

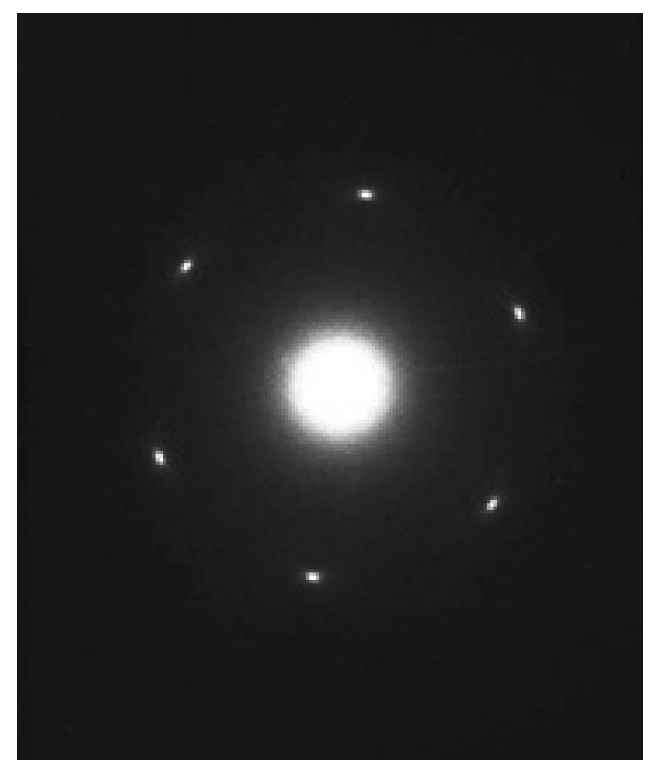

FIG. 4. Electron-diffraction pattern of a six-layer 40.8 film at $47.0{ }^{\circ} \mathrm{C}$. 
the Cry- $B$ phase of 14 S5 than in 4O.8. Mechanical and $\mathrm{x}$-ray measurements in 14S5 to compare with those in $40.8[13,14]$ would be most interesting.

Our results on the two-layer films are of particular interest. Since earlier studies have shown that a two-layer smectic film is effectively $2 \mathrm{D}$ in nature [26,27], it is expected to exhibit only pseudo-long-range positional order in the crystalline phase. Our observation of prominent secondary electron diffraction in a two-layer Cry- $B$ film of 14S5 despite the absence of true long-range positional order is thus rather surprising. Previous $\mathrm{x}$-ray results suggested that the pseudo-long-range positional correlations in thin films, as indicated by the exponent $\eta$, are similar in the 2D Cry- $B$ phase of $14 \mathrm{~S} 5$ and 40.8 $[8,9]$. Nevertheless, we have seen secondary electron diffraction in a two-layer Cry- $B$ film of $14 \mathrm{~S} 5$ but not of 40.8 , suggesting an important difference in the detailed planar positional order in these two $2 \mathrm{D}$ crystals.

We are grateful to Y. H. Liu and Y.C. Shen for participation in the early phase of this work, P. J. Wu for technical improvement in our image analysis and scanning system, and Professor J. M. Cowley, Professor W. H. de Jeu, Professor C. C. Huang, Dr. W. J. Hsieh, Dr. A. J. Jin, and Dr. R. Pindak for helpful discussions. One of us (C.Y.C) expresses thanks to Professor K.T. Liu and Professor C. L. Lin for their encouragement. This work was funded by the National Science Council, Taiwan, Republic of China.

*To whom correspondence should be addressed. Electronic address: cychao@phys.ntu.edu.tw †Current address: Applied NanoBioscience Center, Arizona State University, Tempe, AZ 85287-9409, USA.

[1] F. Bloch, Z. Phys. 61, 206 (1930).

[2] R. E. Peierls, Ann. Inst. Henri Poincaré 5, 177 (1935).

[3] I. Lyuksyutov, A. G. Naumovets, and V. Pokrovsky, Two-Dimensional Crystals (Academic, San Diego, 1992).

[4] T. M. Rice, Phys. Rev. 140, A1889 (1965).
[5] N. D. Mermin, Phys. Rev. 176, 250 (1968).

[6] L. D. Landau and E. M. Lifshitz, Statistical Physics (Addison-Wesley, London, 1969), p. 466.

[7] B. Jancovici, Phys. Rev. Lett. 19, 20 (1967).

[8] D. E. Moncton, R. Pindak, S. C. Davey, and G. S. Brown, Phys. Rev. Lett. 49, 1865 (1982).

[9] D. E. Moncton and R. Pindak, Phys. Rev. Lett. 43, 701 (1979).

[10] D. Y. Noh, J. D. Brock, J. O. Fossum, J. P. Hill, W. J. Nuttall, J. D. Litster, and R. J. Birgeneau, Phys. Rev. B 43, 842 (1991).

[11] R. Pindak, D. J. Bishop, and W. O. Sprenger, Phys. Rev. Lett. 44, 1461 (1980).

[12] D. J. Bishop, W. O. Sprenger, R. Pindak, and M. E. Neubert, Phys. Rev. Lett. 49, 1861 (1982).

[13] M. Cagnon and G. Durand, Phys. Rev. Lett. 45, 1418 (1980).

[14] A. Fera, I. P. Dolbnya, R. Opitz, B. I. Ostrovskii, and W. H. de Jeu, Phys. Rev. E 63, 020601(R) (2001).

[15] C. Y. Chao, C. F. Chou, J. T. Ho, S.W. Hui, A. J. Jin, and C. C. Huang, Phys. Rev. Lett. 77, 2750 (1996).

[16] C. Y. Chao, Y. H. Liu, T. C. Pan, B. N. Chang, and J. T. Ho, Phys. Rev. E 64, 050703(R) (2001).

[17] J. M. Cowley, A. L. G. Rees, and J. A. Spink, Proc. Phys. Soc. London A 64, 609 (1951).

[18] B. K. Vainshtein, Structural Analysis by ElectronDiffraction (Pergamon, Oxford, 1964).

[19] D. L. Dorset, Structural Electron-Diffraction (Plenum, New York, 1995).

[20] M. Cheng, J. T. Ho, S.W. Hui, and R. Pindak, Phys. Rev. Lett. 59, 1112 (1987).

[21] D. R. Nelson and B. I. Halperin, Phys. Rev. B 19, 2457 (1979).

[22] A. P. Young, Phys. Rev. B 19, 1855 (1979).

[23] W. H. de Jeu, B. I. Ostrovkii, and A. N. Shalaginov, Rev. Mod. Phys. 75, 181 (2003).

[24] R. Geer and R. Pindak (unpublished).

[25] S. K. Hark and J. T. Ho, Mol. Cryst. Liq. Cryst. Lett. 56, 99 (1979).

[26] C. F. Chou, J. T. Ho, S. W. Hui, and V. Surendranath, Phys. Rev. Lett. 76, 4556 (1996).

[27] C. F. Chou, J. T. Ho, and S.W. Hui, Phys. Rev. E 56, 592 (1997). 